\title{
A Visualization and a Rating for Naturality on Pairwise Comparisons
}

\author{
Takafumi MIZUNO ${ }^{1}$ \\ Meijo University
}

\begin{abstract}
We have to make a decision even if utility functions and measurement procedures of alternatives are not given clearly. The Analytic Hierarchy Process (AHP) is a multi-criteria decision-making process in transdisciplinary areas. The process does not assume linear order of utility and priorities of alternatives; they are given as ratios from pairwise comparisons with regards on each criterion. This study is on the pairwise comparisons. First, I introduce a criterion, called naturality in this article. It is a generalized concept of Condorcet's principle. And I provide a link diagram to confirm naturality. Finally, I describe a rating procedure to aggregate weights which satisfy naturality.
\end{abstract}

Keywords. Pairwise Comparison, Link Diagram, Category Theory

\section{Introduction}

We often face decision-making problems, which select the best one from the set of alternatives, in social issue or individual's life. It is hard to solve the problems because some criteria conflict with each other, and utility functions or procedures to evaluate alternatives are not always given. We have to make decisions even if suitable disciplines and communications among them are under developing. The Analytic Hierarchy Process (AHP), developed by Saaty [1], is a decision-making process based on pairwise comparisons. If utility functions or any measurements of alternatives are not identified, then pairwise comparisons are the only way to evaluate alternatives. AHP consists of three phases: construction of hierarchy structure, evaluation of weights of alternatives in each criterion by using pairwise comparisons, and synthesis the weights.

This article focuses on pairwise comparisons in the second phase. Each criterion, for every pair of alternatives, decision-makers show the ratio how times prefer the alternative than another one. Weights of alternatives are aggregated from the ratios by using the suitable method.

To describe how to aggregate ratios, let us consider an example in the case of four alternatives $\{1,2,3,4\}$. Ratios given by decision-makers' pairwise comparisons for all pair of alternatives are arranged into the following matrix.

\footnotetext{
${ }^{1}$ Corresponding Author, Email: tmizuno@meijo-u.ac.jp.
} 


$$
A=\left(a_{i j}\right)=\left[\begin{array}{llll}
\frac{1}{1} & 2 & 3 & 2 \\
\frac{1}{2} & 1 & 7 & 5 \\
\frac{1}{3} & \frac{1}{7} & 1 & \frac{1}{2} \\
\frac{1}{2} & \frac{1}{5} & 2 & 1
\end{array}\right]
$$

The element $a_{24}=5$ means that the alternative 2 is 5 times preferred than the alternative 4 . If the alternative $i$ is preferred than $j, i>j$, then $a_{i j}>1$, othewise $a_{i j} \leq$ 1.

In AHP, the weights of alternatives are calculated by the geometric mean method or the eigenvector method. The weights calculated by the geometric mean method are $\left(w_{1}, w_{2}, w_{3}, w_{4}\right)=(0.375,0.412,0.079,0.135)$. They are normalized values of $(\sqrt[4]{12}, \sqrt[4]{35 / 2}, \sqrt[4]{1 / 42}, \sqrt[4]{1 / 5})$ which geometric means of each row of the matrix. The weights calculated by the eigenvector method are $\left(w_{1}, w_{2}, w_{3}, w_{4}\right)=$ $(0.389,0.408,0.075,0.128)$, which are elements of the principal eigenvector of the matrix. Weights of both imply that $2>1>4>3$; the winner is the alternative 2 .

While, the ratios in the matrix show the alternative 1 wins to all of others; $1>2$ $\left(a_{12}=2>1\right), 1>3\left(a_{13}=3>1\right)$, and $1>4\left(a_{14}=2>1\right)$.

We expect that if the alternative $i$ wins to $j$ in an aggregation then $i$ wins to $j$ in pairwise comparison. Above example shows the geometric mean method and the eigenvector method may not satisfies it. In general pairwise comparisons, there is no procedure which satisfies the requirement. So I introduce a modified requirement that is referred to as naturality [2]; if the alternative $i$ wins to $j$ in the aggregation then $i$ must win to $j$ directly or indirectly.

In the following sections, I describe naturality by using functor, and provide a link diagram to visualize naturality. And I provide a rating method to calculate weights which satisfies naturality.

\section{A link diagram visualizing naturality}

Naturality can be described by using terms in category theory [3]; naturality is the existence of covariant functors between path categories. To describe it, I first construct a path category, or often called free category. The category consists of objects and arrows. Each object corresponds to each alternative, and each arrow represents which alternative is preferred in pairwise comparisons. If the alternative $i$ is preferred than $j, i>j$, then the arrow $i \rightarrow j$ exists. If $i$ and $j$ are indifferent, $a_{i j}=1$, then I put conveniently an arrow from the alternative with smaller number to the alternative with larger number; if $a_{i j}=1$ and $i<j$, then there is an arrow $i \rightarrow j$. Composited arrows are also in the category. The arrow $i \rightarrow j$ and the arrow $k \rightarrow l$ can be composited as $i \rightarrow j \rightarrow l$ when $j=k$.

Under the construction, the existence of an arrow from $i$ to $j$ means that the alternative $i$ wins to $j$ directly or indirectly. If the alternative $i$ wins to $j$ directly, then $i \rightarrow j$ exists. If $i$ wins to $j$ indirectly, then there is a composited arrow from $i$ to $j$. If there is the composited arrow is $i \rightarrow p \rightarrow q \rightarrow j$, then $i$ may be defeated by $j$ directly, but $i$ wins to $j$ indirectly; $i>p$ and $p>q$ and $q>j$. 
A category whose arrows represent preferences given from pairwise comparisons is denoted as $\mathcal{L}$, and a category whose arrows represent preferences in an aggregation is denoted as $\mathcal{G}$. Then a correspondence, referred to as covariant functor, from $\mathcal{G}$ to $\mathcal{L}$ is denoted as $F ; F(i)=i$ where $i$ is an object of category, and an arrow $i \rightarrow j$ is corresponded to a arrow from $i$ to $j$ as $F(i \rightarrow j)=i \rightarrow \cdots \rightarrow j$.

If there is a covariant functor $F$ from the category $\mathcal{G}$ which represents the aggregated results to the category $\mathcal{L}$ which represents the pairwise comparisons, then the aggregation satisfies naturality.

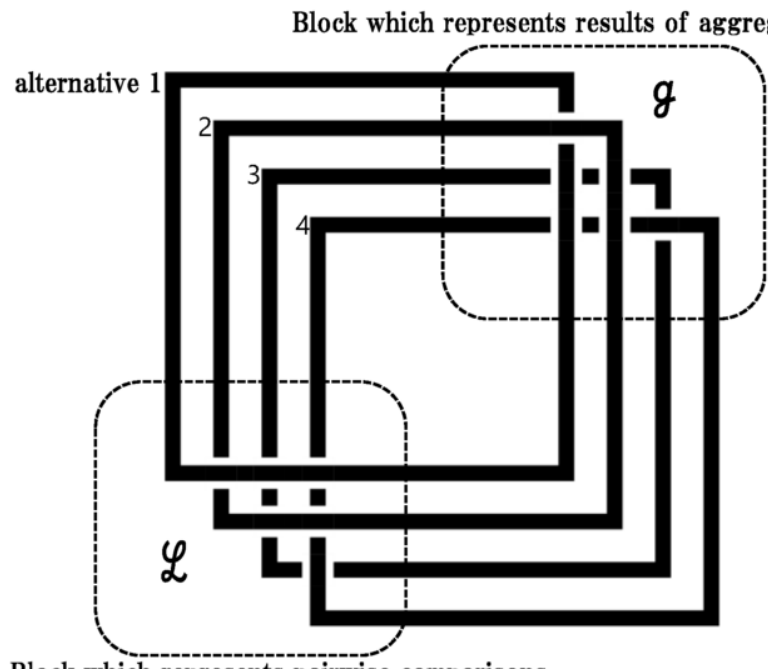

Block which represents pairwise comparisons

Figure 1. A link diagram for the aggregation which does not satisfiy naturallity.

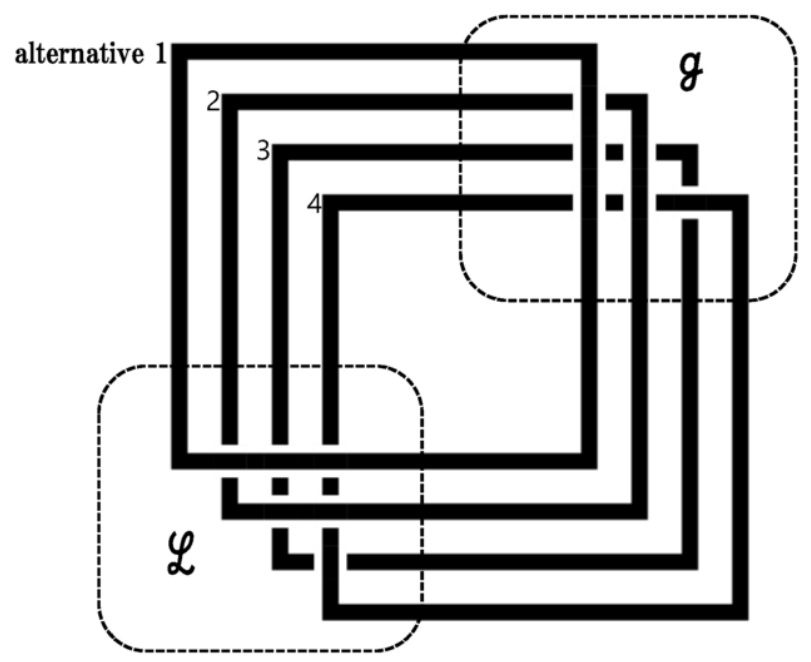

Figure 2. A link diagram for the aggregation which satisfies naturality. 
A link diagram to confirm naturality is provided (Figure 1 and 2) [4]. It consists of loops floating on the ground. Each loop corresponds to each alternative, and crossings of each loop represent which alternative is preferred. If the alternative $i$ wins to $j$ then the loop of $i$ is above the loop of $j$ at the crossing of the two loops. In the diagram, every loop crosses twice other loops. One cross represents a pairwise comparison, and another represents result of an aggregation. Former crossings are in the lower-left side block $\mathcal{L}$ in the diagram, and later are in the upper-right side block $\mathcal{G}$.

An arrow $i \rightarrow j$ of the category is represented as jump from the upper loop $i$ to the lower loop $j$ at the crossing of the two loops. In Figure 1, which visualizes the aggregation of the above example, the arrow $1 \rightarrow 2$ is represented as the jump from the loop of 1 to 2 at the crossing the loop of 1 and 2 . It can jump from 1 to 2 in the block block $\mathcal{G}$, but it cannt jump from 1 to 2 in the block $\mathcal{L}$. It means that it cannot construct any covariant functor; the aggregation does not satisfy naturality. While, in the Figure 2, all jumps in $\mathcal{G}$ have corresponds jumps in $\mathcal{L}$; It satisfies naturality.

The diagram also representes a commutative diagram of a natural transformation $\eta$ from the identity functor $i d$, which changes nothing, to the covariant functor $F$.

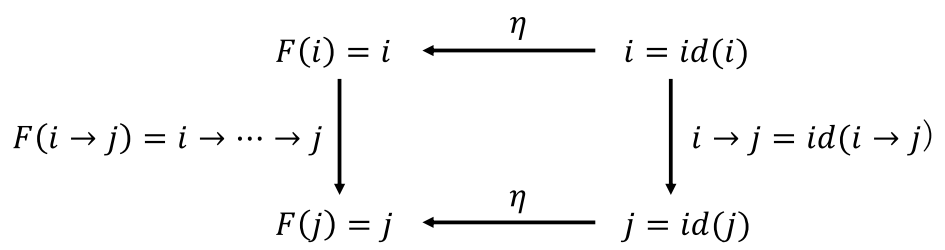

\section{Rating which satisfies naturality}

Each category in this article is equivalence to tournament graph whose nodes and edges correspond objects and arrows of the category, respectively. From the graph, we can extract Hamilton path [5], which is a directed path that visits each node exactly once. It is clear that the order of nodes in the path satisfies naturality.

There are many procedures to extract Hamilton paths from tournament graph. Let us suppose that the path is $1 \rightarrow 2 \rightarrow \cdots \rightarrow n$, and consider how to estimate weights of alternatives on the order. It is clear that the weights hold $w_{i} \geq w_{i+1}$. While, the weight $w_{i}$ are estimated from $w_{j}$ as $w_{i}=a_{i j} w_{j}, i \neq j$. So, I provide the rating method to calculate weights as $w_{n}=1$, and $w_{n-1}=a_{n-1, n} w_{n}$, and $w_{i}=$ $\left(a_{i, i+1} w_{i+1} \prod_{j=i+2}^{n} \max \left\{w_{i+1}, a_{i j} w_{j}\right\}\right)^{\frac{1}{n-i}}$, for $i=n-2, \ldots, 1$. Since $a_{i, i+1} \geq 1$, the weights satisfies $w_{i} \geq w_{i+1}$. The weights are decided sequentially from $w_{n}$ to $w_{1}$. Each weight is calculated as geometric mean among estimated weights from weights already decided. If the estimated weights $a_{i j} w_{j}, j>i+1$, is less than $w_{i+1}$, then it is replaced to $w_{i+1}$ to satisfy the inequality.

For the above example, the Hamilton path $1 \rightarrow 2 \rightarrow 4 \rightarrow 3$ can be extracted. On the path, the rating method calculates the weights as follows: 


$$
\begin{aligned}
& w_{1}=\left(a_{12} w_{2} \max \left\{w_{2}, a_{14} w_{4}\right\} \max \left\{w_{2}, a_{13} w_{3}\right\}\right)^{\frac{1}{3}}=10.542, \\
& w_{2}=\left(a_{24} w_{4} \max \left\{w_{4}, a_{23} w_{3}\right\}\right)^{\frac{1}{2}}=(10 \max \{2,7\})^{\frac{1}{2}}=8.367, \\
& w_{3}=1.000, \\
& w_{4}=a_{43} w_{3}=2.000,
\end{aligned}
$$

or $(0.481,0.382,0.046,0.091)$ with normalizing them. And also the aggregation is visualized in Figure 2.

Many rating method can be considered. Mizuno [2] has provided simple rating method to decide weights as follows; $w_{n}=1, w_{n-1}=a_{n-1, n} w_{n}$, and $w_{i}=a_{i, i+1} w_{i+1}$.

$$
\begin{aligned}
w_{1} & =a_{12} a_{23} a_{34} \cdots a_{n-1, n} \\
w_{2} & =a_{23} a_{34} \cdots a_{n-1, n} \\
& \vdots \\
w_{n-1} & =a_{n-1, n} \\
w_{n} & =1 .
\end{aligned}
$$

It satisfies $w_{i} / w_{i+1}=a_{i, i+1}$ for all $i=1, \ldots, n-1$. The rating satisfies naturality when the Hamilton path is $1 \rightarrow 2 \rightarrow \cdots \rightarrow n$, and also the weights have following property:

$$
\left|\frac{w_{i}}{w_{i+1}}-a_{i, i+1}\right|=0, \quad i=1, \ldots, n-1 .
$$

The rating is just ignore some pairwise comparisons, but the weights are optimal; decreasing $\left|w_{j} / w_{k}-a_{j k}\right|, k \neq j$ and $k \neq j+1$, increases $\left|w_{i} / w_{i+1}-a_{i, i+1}\right|$ for some $i$.

\section{Conclusions}

I provided a link diagram that visualizes a criterion, naturality, on pairwise comparisons. The diagram represents natural transformations between identity functor and covariant functor. And I provided a rating method to calculate weights of alternatives. The method aggregated weights which satisfy naturality.

If there is no cycle of preference in pairwise comparisons, then loops in the link diagram which satisfies naturality are splittable.

Naturality is a generalization of Condorcet's principle [6] which states the alternative which wins to all of the others in pairwise comparisons must be the winner of the aggregation when such alternative exists.

\section{References}

[1] Saaty, T.L., The Analytic Hierarchy Process, McGraw Hill, New York, 1980.

[2] Mizuno, T., Naturality for Ranking from Pairwise Comparisons, Intelligent Decision Technologies 2019, Smart Innovation, Systems and Technologies, under printing.

[3] Leinster, T., Basic Category Theory, Cambridge University Press, Cambridge, 2014.

[4] Mizuno, T., A Link Diagram for Pairwise Comparisons, Intelligent Decision Technologies 2018, Smart Innovation, Systems and Technologies, pp.181-186, Springer, 2019.

[5] Wilson, R.J., Introduction to Graph Theory, Pearson, Harlow 2012. 
[6] Condorcet, M., Essai sur l'application de l'analyse a la probabilite des decisions rendues a la pluralite des voix, de l'Imprimerie royale, Paris, 1785. 